\title{
ON THE CESÀRO SUMMABILITY FOR FUNCTIONS OF TWO VARIABLES
}

\section{Ü. TOTUR AND İ. ÇANAK}

Received 06 November, 2016

Abstract. For a continuous function $f(T, S)$ on $\mathbb{R}_{+}^{2}=[0, \infty) \times[0, \infty)$, we define its integral on $\mathbb{R}_{+}^{2}$ by

and its $(C, \alpha, \beta)$ mean by

$$
F(T, S)=\int_{0}^{T} \int_{0}^{S} f(t, s) d t d s
$$

$$
\sigma_{\alpha, \beta}(T, S)=\int_{0}^{T} \int_{0}^{S}\left(1-\frac{t}{T}\right)^{\alpha}\left(1-\frac{s}{S}\right)^{\beta} f(t, s) d t d s,
$$

where $\alpha>-1$, and $\beta>-1$. We say that $\int_{0}^{\infty} \int_{0}^{\infty} f(t, s) d t d s$ is $(C, \alpha, \beta)$ integrable to $L$ if $\lim _{T, S \rightarrow \infty} \sigma_{\alpha, \beta}(T, S)=L$ exists.

We prove that if $\lim _{T, S \rightarrow \infty} \sigma_{\alpha, \beta}(T, S)=L$ exists for some $\alpha>-1$ and $\beta>-1$, then $\lim _{T, S \rightarrow \infty} \sigma_{\alpha+h, \beta+k}(T, S)=L$ exists for all $h>0$ and $k>0$.

Next, we prove that if $\int_{0}^{\infty} \int_{0}^{\infty} f(t, s) d t d s$ is $(C, 1,1)$ integrable to $L$ and

$$
T \int_{0}^{S} f(T, s) d s=O(1)
$$

and

$$
S \int_{0}^{T} f(t, S) d s=O(1)
$$

then $\lim _{T, S \rightarrow \infty} F(T, S)=L$ exists.

2010 Mathematics Subject Classification: 40A10; 40C10; 40D05; 40G05

Keywords: $(C, \alpha, \beta)$ integrability, improper double integral, convergence in Pringsheim's sense, Cesàro integrability $(C, 1,1),(C, 1,0)$ and $(C, 0,1)$, Tauberian conditions and theorems

\section{INTRODUCTION}

Let $f(t)$ be a continuous function on $[0, \infty)$. The improper integral $\int_{0}^{\infty} f(t) d t$ is said to be $(C, \alpha)$ integrable to $L$ for some $\alpha>-1$ if the limit

$$
\lim _{T \rightarrow \infty} \int_{0}^{T}\left(1-\frac{t}{T}\right)^{\alpha} f(t) d t=L
$$

(c) 2018 Miskolc University Press 
exists. For all $\alpha, \beta \in \mathbb{R}$ with $-1<\alpha<\beta$, the $(C, \alpha)$ integrability implies the $(C, \beta)$ integrability. This implication is a classical result in the summability theory $[1, \mathrm{p}$. 106]. The converse of this implication may be true by adding some suitable condition on the $(C, \beta)$ integrability of the improper integral $\int_{0}^{\infty} f(t) d t$. Any theorem which states that convergence of the improper integral follows from the $(C, \alpha)$ integrability of the improper integral and a Tauberian condition is said to be a Tauberian theorem.

As a special case, Laforgia [7] obtained a sufficient condition under which convergence of the improper integral follows from $(C, 1)$ integrability of the improper integral. Móricz and Németh [9] established some one-sided and two-sided bounded Tauberian conditions for real or complex valued functions. Recently, Çanak and Totur $([2,3])$ have proved the generalized Littlewood theorem and Hardy-Littlewood type Tauberian theorems for the $(C, 1)$ integrability of a continuous function on $[0, \infty)$ by using the concept of the general control modulo analogous to the one defined by Dik [6]. Çanak and Totur [4] have also given alternative proofs of some classical type Tauberian theorems for the $(C, 1)$ integrability of a continuous function on $[0, \infty)$. Çanak and Totur [5] generalized the results of Laforgia [7] for the $(C, \alpha)$ integrability of functions by weighted mean methods.

For a continuous function $f(T, S)$ on $\mathbb{R}_{+}^{2}=[0, \infty) \times[0, \infty)$, we define its integral on $\mathbb{R}_{+}^{2}$ by

$$
F(T, S)=\int_{0}^{T} \int_{0}^{S} f(t, s) d t d s,
$$

and its $(C, \alpha, \beta)$ mean by

$$
\sigma_{\alpha, \beta}(T, S)=\int_{0}^{T} \int_{0}^{S}\left(1-\frac{t}{T}\right)^{\alpha}\left(1-\frac{s}{S}\right)^{\beta} f(t, s) d t d s,
$$

where $\alpha>-1$ and $\beta>-1$. An improper integral

$$
\int_{0}^{\infty} \int_{0}^{\infty} f(t, s) d t d s
$$

is said to be $(C, \alpha, \beta)$ integrable to $L$ if

$$
\lim _{T, S \rightarrow \infty} \sigma_{\alpha, \beta}(T, S)=L
$$

The $(C, 0,0)$ integrability is the convergence of the improper integral (1.2).

However, there are some $(C, \alpha, \beta)$ integrable functions which fail to converge as improper integrals. Adding some Tauberian condition, one may get the converse.

In this paper, we prove that the $(C, \alpha, \beta)$ integrability of (1.2) where $\alpha>-1$ and $\beta>-1$ implies the $(C, \alpha+h, \beta+k)$ integrability of (1.2) for all $h>0$ and $k>0$. As a corollary to this result, we show that if $(1.2)$ converges to $L$, then $(1.2)$ is $(C, h, k)$ integrable to $L$ for all $h>0$ and $k>0$. But, the converse of this implication might be true under some conditions imposed on the function. Furthermore, we give conditions under which (1.2) follows from the $(C, 1,1)$ integrability of $(1.2)$. It will be shown as 
a corollary of our first result in this paper that convergence of the improper integral (1.2) implies the existence of the $\operatorname{limit}_{T, S \rightarrow \infty} \sigma_{h, k}(T, S)$ for all $h>0$ and $k>0$.

\section{RESULTS}

The following theorem shows that $(C, \alpha, \beta)$ integrability of (1.2), where $\alpha>-1$ and $\beta>-1$, implies $(C, \alpha+h, \beta+k)$ integrability of (1.2), where all $h>0$ and $k>0$.

Theorem 1. If (1.2) is $(C, \alpha, \beta)$ integrable to $L$ for some $\alpha>-1$ and $\beta>-1$, then it is $(C, \alpha+h, \beta+k)$ integrable to $L$ for all $h>0$ and $k>0$.

Proof. Consider

$$
\int_{0}^{T} \int_{0}^{S} \varphi(t, s ; T, S) \sigma_{\alpha, \beta}(T, S) d t d s
$$

where

$$
\varphi(t, s ; T, S)=\frac{1}{B(\alpha+1, h)} \frac{1}{B(\beta+1, k)} \frac{1}{T}\left(\frac{t}{T}\right)^{\alpha}\left(1-\frac{t}{T}\right)^{h-1} \frac{1}{S}\left(\frac{s}{S}\right)^{\beta}\left(1-\frac{s}{S}\right)^{k-1}
$$

where $B$ denotes the Beta function defined by

$$
B(x, y)=\int_{0}^{1} t^{x-1}(1-t)^{y-1} d t, \quad x>0, y>0 .
$$

Letting $u=\frac{t}{T}$ and $v=\frac{s}{S}$, we have

$$
\int_{0}^{T} \int_{0}^{S} \varphi(t, s ; T, S) d t d s=1
$$

We need to prove that

$$
\lim _{T, S \rightarrow \infty} \int_{0}^{T} \int_{0}^{S} \varphi(t, s ; T, S) \sigma_{\alpha, \beta}(T, S) d t d s=L .
$$

Since

$$
\lim _{T, S \rightarrow \infty} \sigma_{\alpha, \beta}(T, S)=L
$$

by the hypothesis, there exists a value $T_{\varepsilon}$ for any given $\varepsilon>0$ such that

$$
\left|\sigma_{\alpha, \beta}(T, S)-L\right|<\varepsilon, \quad T \geq T_{\varepsilon}, S \geq S_{\varepsilon} .
$$

It follows from (2.3) that

$$
\begin{aligned}
& \int_{0}^{T} \int_{0}^{S} \varphi(t, s ; T, S) \sigma_{\alpha, \beta}(T, S) d t d s-L \\
&=\int_{0}^{T} \int_{0}^{S} \varphi(t, s ; T, S)\left[\sigma_{\alpha, \beta}(T, S)-L\right] d t d s
\end{aligned}
$$


To prove (2.4), it suffices to show that

$$
\left|\int_{0}^{T} \int_{0}^{S} \varphi(t, s ; T, S) \sigma_{\alpha, \beta}(T, S) d t d s-L\right|<4 \varepsilon,
$$

provided that $T$ and $S$ are large enough.

We notice that by the hypothesis, the function $\sigma_{\alpha, \beta}(T, S)$ is bounded on $\mathbb{R}_{+}^{2}$, that is,

$$
\left|\sigma_{\alpha, \beta}(T, S)-L\right|<K, \quad 0 \leq T, S<\infty,
$$

for some constant $K$. Using (2.3) and (2.6), we obtain, by (2.7),

$$
\begin{aligned}
& \left|\int_{0}^{T} \int_{0}^{S} \varphi(t, s ; T, S)\left[\sigma_{\alpha, \beta}(T, S)-L\right] d t d s\right| \\
& \leq \int_{0}^{T_{\epsilon}} \int_{0}^{S_{\epsilon}} \varphi(t, s ; T, S)\left|\sigma_{\alpha, \beta}(T, S)-L\right| d t d s \\
& \quad+\int_{0}^{T_{\epsilon}} \int_{S_{\epsilon}}^{S} \varphi(t, s ; T, S)\left|\sigma_{\alpha, \beta}(T, S)-L\right| d t d s \\
& \quad+\int_{T_{\epsilon}}^{T} \int_{0}^{S_{\epsilon}} \varphi(t, s ; T, S)\left|\sigma_{\alpha, \beta}(T, S)-L\right| d t d s \\
& \quad+\epsilon \int_{T_{\epsilon}}^{T} \int_{S_{\epsilon}}^{S} \varphi(t, s ; T, S) d t d s \\
& \leq K \int_{0}^{T_{\epsilon}} \int_{0}^{S_{\epsilon}} \varphi(t, s ; T, S) d t d s+K \int_{0}^{T_{\epsilon}} \int_{S_{\epsilon}}^{S} \varphi(t, s ; T, S) d t d s \\
& \quad+K \int_{T_{\epsilon}}^{T} \int_{0}^{S_{\epsilon}} \varphi(t, s ; T, S) d t d s+\epsilon \int_{0}^{T} \int_{0}^{S} \varphi(t, s ; T, S) d t d s \\
& =K \int_{0}^{T_{\epsilon}} \int_{0}^{S_{\epsilon}} \varphi(t, s ; T, S) d t d s+K \int_{0}^{T_{\epsilon}} \int_{S_{\epsilon}}^{S} \varphi(t, s ; T, S) d t d s \\
& \quad+K \int_{T_{\epsilon}}^{T} \int_{0}^{S_{\epsilon}} \varphi(t, s ; T, S) d t d s+\epsilon
\end{aligned}
$$

By the substitution $u=\frac{t}{T}, v=\frac{s}{S}$, we have

$$
\begin{aligned}
\int_{0}^{T_{\epsilon}} \int_{0}^{S_{\epsilon}} \varphi(t, s ; T, S) d t d s= & \frac{1}{B(\alpha+1, h)} \int_{0}^{T_{\varepsilon}} \frac{1}{T}\left(\frac{t}{T}\right)^{\alpha}\left(1-\frac{t}{T}\right)^{h-1} d t \\
& \times \frac{1}{B(\beta+1, k)} \int_{0}^{S_{\epsilon}} \frac{1}{S}\left(\frac{s}{S}\right)^{\beta}\left(1-\frac{s}{S}\right)^{k-1} d s
\end{aligned}
$$




$$
\begin{aligned}
= & \frac{1}{B(\alpha+1, h)} \int_{0}^{T_{\varepsilon} / T} u^{\alpha}(1-u)^{h-1} d u \\
& \times \frac{1}{B(\beta+1, k)} \int_{0}^{S_{\varepsilon} / S} v^{\beta}(1-v)^{k-1} d v
\end{aligned}
$$

which tends to zero when $T, S \rightarrow \infty$ for any fixed $T_{\varepsilon}$ and $S_{\varepsilon}$. Thus, there exist some $\widehat{T_{\varepsilon}^{1}}$ and $\widehat{S_{\varepsilon}^{1}}$ such that

$$
K \int_{0}^{T_{\epsilon}} \int_{0}^{S_{\epsilon}} \varphi(t, s ; T, S) d t d s<\varepsilon, \quad T \geq \widehat{T_{\varepsilon}^{1}}, S \geq \widehat{S_{\varepsilon}^{1}}
$$

By the substitution $u=\frac{t}{T}, v=\frac{s}{S}$, we have

$$
\begin{aligned}
\int_{0}^{T_{\epsilon}} \int_{S_{\epsilon}}^{S} \varphi(t, s ; T, S) d t d s= & \frac{1}{B(\alpha+1, h)} \int_{0}^{T_{\varepsilon}} \frac{1}{T}\left(\frac{t}{T}\right)^{\alpha}\left(1-\frac{t}{T}\right)^{h-1} d t \\
& \times \frac{1}{B(\beta+1, k)} \int_{S_{\epsilon}}^{S} \frac{1}{S}\left(\frac{S}{S}\right)^{\beta}\left(1-\frac{s}{S}\right)^{k-1} d s \\
= & \frac{1}{B(\alpha+1, h)} \int_{0}^{T_{\varepsilon} / T} u^{\alpha}(1-u)^{h-1} d u \\
& \times \frac{1}{B(\beta+1, k)} \int_{S_{\varepsilon} / S}^{1} v^{\beta}(1-v)^{k-1} d v
\end{aligned}
$$

which tends to zero when $T, S \rightarrow \infty$ for any fixed $T_{\varepsilon}$ and $S_{\varepsilon}$ (Note that $\frac{1}{B(\beta+1, k)} \int_{S_{\varepsilon} / S}^{1} v^{\beta}(1-v)^{k-1} d v$ tends to 1 as $\left.S \rightarrow \infty\right)$. Thus, there exist some $\widehat{T_{\varepsilon}^{2}}$ and $\widehat{S_{\varepsilon}^{2}}$ such that

$$
K \int_{0}^{T_{\epsilon}} \int_{S_{\epsilon}}^{S} \varphi(t, s ; T, S) d t d s<\varepsilon, T \geq \widehat{T_{\varepsilon}^{2}}, S \geq \widehat{S_{\varepsilon}^{2}} .
$$

Similarly, the integral

$$
\int_{T_{\epsilon}}^{T} \int_{0}^{S_{\epsilon}} \varphi(t, s ; T, S) d t d s
$$

tends to to zero when $T, S \rightarrow \infty$ for any fixed $T_{\varepsilon}$ and $S_{\varepsilon}$ (Note that $\frac{1}{B(\alpha+1, h)} \int_{T_{\varepsilon} / T}^{1} u^{\alpha}(1-u)^{k-1} d v$ tends to 1 as $\left.S \rightarrow \infty\right)$. Thus, there exist some $\widehat{T_{\varepsilon}^{3}}$ and $\widehat{S_{\varepsilon}^{3}}$ such that

$$
K \int_{T_{\epsilon}}^{T} \int_{0}^{S_{\epsilon}} \varphi(t, s ; T, S) d t d s<\varepsilon, \quad T \geq \widehat{T_{\varepsilon}^{3}}, S \geq \widehat{S_{\varepsilon}^{3}}
$$


Hence, we have (2.8) for $T \geq \max \left\{T_{\epsilon}, \widehat{T}_{\varepsilon}^{1},{\widehat{T_{\varepsilon}}}^{2}, \widehat{T}_{\varepsilon}^{3}\right\}, S \geq \max \left\{S_{\epsilon},{\widehat{S_{\varepsilon}}}^{1},{\widehat{S_{\varepsilon}}}^{2},{\widehat{S_{\varepsilon}}}^{3}\right\}$ and this proves (2.4). We obtain

$$
\begin{aligned}
& \int_{0}^{T} \int_{0}^{S} \varphi(t, s ; T, S) \sigma_{\alpha, \beta}(t, s) d t d s \\
& =\int_{0}^{T} \int_{0}^{S} \varphi(t, s ; T, S)\left(\int_{0}^{t} \int_{0}^{s}\left(1-\frac{u}{t}\right)^{\alpha}\left(1-\frac{v}{s}\right)^{\beta} f(u, v) d u d v\right) d t d s \\
& =\int_{0}^{T} \int_{0}^{S} f(u, v)\left(\int_{u}^{T} \int_{v}^{S} \varphi(t, s ; T, S)\left(1-\frac{u}{t}\right)^{\alpha}\left(1-\frac{v}{s}\right)^{\beta} d t d s\right) d u d v \\
& =\int_{0}^{T} \int_{0}^{S} f(u, v) I(u, v ; T, S) d u d v,
\end{aligned}
$$

where

$$
I(u, v ; T, S)=\int_{u}^{T} \int_{v}^{S} \varphi(t, s ; T, S)\left(1-\frac{u}{t}\right)^{\alpha}\left(1-\frac{v}{S}\right)^{\beta} d t d s .
$$

Here, we write $I(u, v ; T, S)$ as

$$
\begin{aligned}
I(u, v ; T, S)= & \int_{u}^{T} \int_{v}^{S} \varphi(t, s ; T, S)\left(1-\frac{u}{t}\right)^{\alpha}\left(1-\frac{v}{s}\right)^{\beta} d t d s \\
= & \left(\frac{1}{B(\alpha+1, h)} \int_{u}^{T} \frac{1}{T}\left(\frac{t}{T}\right)^{\alpha}\left(1-\frac{t}{T}\right)^{h-1}\left(1-\frac{u}{t}\right)^{\alpha} d t\right) \\
& \times\left(\frac{1}{B(\beta+1, k)} \int_{v}^{S} \frac{1}{S}\left(\frac{S}{S}\right)^{\beta}\left(1-\frac{s}{S}\right)^{k-1}\left(1-\frac{v}{s}\right)^{\beta} d s\right) \\
= & \left(\frac{1}{B(\alpha+1, h)}\left(\frac{1}{T}\right)^{\alpha+1} \int_{u}^{T}\left(1-\frac{t}{T}\right)^{h-1}(t-u)^{\alpha} d t\right) \\
& \times\left(\frac{1}{B(\beta+1, k)}\left(\frac{1}{S}\right)^{\beta+1} \int_{v}^{S}\left(1-\frac{s}{S}\right)^{k-1}(s-v)^{\beta} d s\right) \\
= & I_{1}(u, T) I_{2}(v, S),
\end{aligned}
$$

where

$$
I_{1}(u, T)=\frac{1}{B(\alpha+1, h)}\left(\frac{1}{T}\right)^{\alpha+1} \int_{u}^{T}\left(1-\frac{t}{T}\right)^{h-1}(t-u)^{\alpha} d t
$$

and

$$
I_{2}(v, S)=\frac{1}{B(\beta+1, k)}\left(\frac{1}{S}\right)^{\beta+1} \int_{v}^{S}\left(1-\frac{s}{S}\right)^{k-1}(s-v)^{\beta} d s .
$$


Substituting $t=T-(T-u) x$ in $I_{1}(u, T)$, we have

$$
\begin{aligned}
I_{1}(u, T) & =\frac{1}{B(\alpha+1, h)}\left(1-\frac{u}{T}\right)^{h-1}\left(1-\frac{u}{T}\right)^{\alpha+1} \int_{0}^{1} x^{h-1}(1-x)^{\alpha} d x \\
& =\left(1-\frac{u}{T}\right)^{\alpha+h},
\end{aligned}
$$

and similarly we have

$$
I_{2}(v, S)=\left(1-\frac{v}{S}\right)^{\beta+k}
$$

These show that

$$
\begin{aligned}
& \int_{0}^{T} \int_{0}^{S} \varphi(t, s ; T, S) \sigma_{\alpha, \beta}(t, s) d t d s \\
& =\int_{0}^{T} \int_{0}^{S}\left(1-\frac{u}{T}\right)^{\alpha+h}\left(1-\frac{v}{S}\right)^{\beta+k} f(u, v) d u d v=\sigma_{\alpha+h, \beta+k}(T, S)
\end{aligned}
$$

This completes the proof of Theorem 1 .

\section{THE CASE $\alpha=1, \beta=0$ OR $\alpha=0, \beta=1$}

Similar to the $(C, 1,1)$ integrability, one can improve the theory of the $(C, 1,0)$ or the $(C, 0,1)$ integrability. Since this theory is similar to the theory of integrability of functions of one variable, we only present it without detailed proofs.

Definition 1. Let $f(T, S)$ be a continuous function on $\mathbb{R}_{+}^{2}$ and $F(T, S)$ be defined as in $(1.1)$. We define $(C, 1,0)$ and $(C, 0,1)$ means of $(1.1)$ by

$$
\sigma_{1,0}(T, S):=\int_{0}^{T} \int_{0}^{S}\left(1-\frac{t}{T}\right) f(t, s) d t d s
$$

and

$$
\sigma_{0,1}(T, S):=\int_{0}^{T} \int_{0}^{S}\left(1-\frac{s}{S}\right) f(t, s) d t d s,
$$

respectively. We say that $(1.2)$ is $(C, 1,0)$ and $(C, 0,1)$ integrable on $\mathbb{R}_{+}^{2}$ if

$$
\lim _{T, S \rightarrow \infty} \sigma_{1,0}(T, S)
$$

and

$$
\lim _{T, S \rightarrow \infty} \sigma_{0,1}(T, S)
$$

exist and are finite, respectively.

The $(C, 1,0)$ and $(C, 0,1)$ summability methods are regular method. Namely, if (1.2) converges to $L$, then $(1.2)$ is both $(C, 1,0)$ and $(C, 0,1)$ integrable to $L$. 
Theorem 2. If $(1.2)$ is $(C, 1,0)$ integrable to $L$ and

$$
T \int_{0}^{S} f(T, s) d s=O(1)
$$

then (1.2) converges to $L$.

Theorem 3. If $(1.2)$ is $(C, 0,1)$ integrable to $L$

$$
S \int_{0}^{T} f(t, S) d t=O(1)
$$

then (1.2) converges to $L$.

Since the proofs of Theorem 2 and Theorem 3 can be obtained with similar steps as in Theorem 3.2 in [7], we omit the proofs.

\section{The CASE $\alpha=1, \beta=1$}

Definition 2. Let $f(T, S)$ be a continuous function on $\mathbb{R}_{+}^{2}$. We say that (1.2) is $(C, 1,1)$ integrable on $\mathbb{R}_{+}^{2}$, if

$$
\lim _{T, S \rightarrow \infty} \int_{0}^{T} \int_{0}^{S}\left(1-\frac{t}{T}\right)\left(1-\frac{s}{S}\right) f(t, s) d t d s
$$

exists and is finite.

As a result of Theorem 1, we have the following corollary.

Corollary 1. If (1.2) converges to $L$, then $(1.2)$ is $(C, 1,1)$ integrable to $L$.

Proof. Take $\alpha=\beta=0$ and $h=k=1$ in Theorem 1.

That the converse of Corollary 1 is not true in general is shown by the following examples.

Example 1. The integral $\int_{0}^{\infty} \int_{0}^{\infty} \cos t \cos s d t d s$ converges to zero, in $(C, 1,1)$ sense.

By (4.1), we have

$$
\begin{aligned}
& \int_{0}^{T} \int_{0}^{S}\left(1-\frac{t}{T}\right)\left(1-\frac{s}{S}\right) \cos t \cos s d t d s \\
& =\int_{0}^{T}\left(1-\frac{t}{T}\right) \cos t d t \int_{0}^{S}\left(1-\frac{s}{S}\right) \cos s d s=\frac{(1-\cos T)(1-\cos S)}{T S},
\end{aligned}
$$

which tends to zero as $T, S \rightarrow \infty$.

Example 2. The integral $\int_{0}^{\infty} \int_{0}^{\infty} \sin t \sin s d t d s$ converges to 1 , in $(C, 1,1)$ sense. 
By (4.1), we have

$$
\begin{aligned}
& \int_{0}^{T} \int_{0}^{S}\left(1-\frac{t}{T}\right)\left(1-\frac{s}{S}\right) \sin t \sin s d t d s \\
& =\int_{0}^{T}\left(1-\frac{t}{T}\right) \sin t d t \int_{0}^{S}\left(1-\frac{s}{S}\right) \sin s d s=\frac{(T-\sin T)(S-\sin S)}{T S},
\end{aligned}
$$

which tends to 1 as $T, S \rightarrow \infty$.

A convolution theorem for $(C, 1,1)$ integrability is given by the following theorem.

Theorem 4. Let the integrals

$$
\int_{0}^{\infty} \int_{0}^{\infty} f(t, s) d t d s, \int_{0}^{\infty} \int_{0}^{\infty} g(t, s) d t d s
$$

be convergent in $(C, 0,0)$ sense, to $L_{1}$ and $L_{2}$, respectively. Then

$$
h(t, s)=\int_{0}^{t} \int_{0}^{s} f(t-u, s-v) g(t, s) d u d v
$$

converges in $(C, 1,1)$ sense to $L_{1} L_{2}$.

Proof. We need to show that

$$
\lim _{T, S \rightarrow \infty} \int_{0}^{T} \int_{0}^{S}\left(1-\frac{t}{T}\right)\left(1-\frac{s}{S}\right) h(t, s) d t d s=L_{1} L_{2}
$$

We define $F$ and $G$ by

$$
F(t, s)=\int_{0}^{t} \int_{0}^{s} f(t, s) d t d s
$$

and

$$
G(t, s)=\int_{0}^{t} \int_{0}^{s} g(t, s) d t d s
$$

By (4.2), we get

$$
\begin{aligned}
& \int_{0}^{T} \int_{0}^{S}\left(1-\frac{t}{T}\right)\left(1-\frac{s}{S}\right) h(t, s) d t d s \\
& =\int_{0}^{T} \int_{0}^{S}\left(1-\frac{t}{T}\right)\left(1-\frac{s}{S}\right)\left(\int_{0}^{t} \int_{0}^{s} f(t-u, s-v) g(t, s) d u d v\right) d t d s \\
& =\int_{0}^{T} \int_{0}^{S} g(t, s) d u d v\left(\int_{u}^{T} \int_{v}^{S}\left(1-\frac{t}{T}\right)\left(1-\frac{s}{S}\right) f(t-u, s-v) d t d s\right)
\end{aligned}
$$

The substitutions $t-u=\omega$ and $s-v=\delta$ and the subsequent integration by parts give

$$
\int_{0}^{T} \int_{0}^{S} g(t, s) d u d v \int_{u}^{T} \int_{v}^{S}\left(1-\frac{t}{T}\right)\left(1-\frac{s}{S}\right) f(t-u, s-v) d t d s
$$




$$
\begin{aligned}
& =\int_{0}^{T} \int_{0}^{S} g(u, v) d u d v \int_{0}^{T-u} \int_{0}^{S-v}\left(1-\frac{u+\omega}{T}\right)\left(1-\frac{v+\delta}{S}\right) f(\omega, \delta) d \omega d \delta \\
& =\frac{1}{T S} \int_{0}^{T} \int_{0}^{S} G(u, v) F(T-u, S-v) d u d v
\end{aligned}
$$

Since $F(T, S) \rightarrow L_{1}$ and $G(T, S) \rightarrow L_{2}$ as $T \rightarrow \infty$ and $S \rightarrow \infty$, we have that for some $T_{\epsilon}$ and $S_{\epsilon}$

$$
\left|F(T, S)-L_{1}\right| \leq \epsilon
$$

and

$$
\left|G(T, S)-L_{2}\right| \leq \epsilon
$$

for $T \geq T_{\epsilon}$ and $S \geq S_{\epsilon}$. Then we have

$$
\begin{aligned}
& \left|\frac{1}{T S} \int_{0}^{T} \int_{0}^{S} G(u, v) F(T-u, S-v) d u d v-L_{1} L_{2}\right| \\
& =\mid \frac{1}{T S} \int_{0}^{T} \int_{0}^{S}\left(G(u, v)-L_{2}\right) F(T-u, S-v) d u d v \\
& \quad+\int_{0}^{T} \int_{0}^{S} L_{2}\left(F(T-u, S-v)-L_{1}\right) d u d v \mid \\
& \leq \frac{1}{T S} \int_{0}^{T_{\epsilon}} \int_{0}^{S_{\epsilon}}\left|L_{2}\right|\left|F(T-u, S-v)-L_{1}\right| d u d v \\
& \quad+\frac{1}{T S} \int_{0}^{T_{\epsilon}} \int_{S_{\epsilon}}^{S}\left|L_{2}\right|\left|F(T-u, S-v)-L_{1}\right| d u d v \\
& \quad+\frac{1}{T S} \int_{T_{\epsilon}}^{T} \int_{0}^{S_{\epsilon}}\left|L_{2}\right|\left|F(T-u, S-v)-L_{1}\right| d u d v \\
& \quad+\frac{1}{T S} \int_{T_{\epsilon}}^{T} \int_{S_{\epsilon}}^{S}\left|L_{2}\right|\left|F(T-u, S-v)-L_{1}\right| d u d v
\end{aligned}
$$

Since $F(T, S) \rightarrow L_{1}$ and $G(T, S) \rightarrow L_{2}$ as $T \rightarrow \infty$ and $S \rightarrow \infty$, there exist some constants $N_{1}$ and $N_{2}$ such that

$$
\left|F(T, S)-L_{1}\right| \leq N_{1}
$$

and

$$
\left|G(T, S)-L_{2}\right| \leq N_{2}
$$

for all $T$ and $S$. If we use (4.6), (4.7), (4.8), and (4.9), and then letting $T$ and $S$ tend to $\infty$ independently, we have the desired result.

By the next theorem we give a sufficient condition under which $(C, 1,1)$ integrability of (1.2) follows from $(C, 0,0)$ integrability of (1.2). 
Theorem 5. If $(1.2)$ is $(C, 1,1)$ integrable to $L$ and

$$
T \int_{0}^{S} f(T, s) d s=O(1)
$$

and

then (1.2) converges to $L$.

$$
S \int_{0}^{T} f(t, S) d t=O(1)
$$

Proof. Let (1.2) be $(C, 1,1)$ integrable to $L$; that is,

$$
G(T, S):=\int_{0}^{T} \int_{0}^{S}\left(1-\frac{t}{T}\right)\left(1-\frac{s}{S}\right) f(t, s) d t d s \rightarrow L, T, S \rightarrow \infty
$$

We rewrite $G(T, S)$ as

$$
G(T, S)=\int_{0}^{T}\left(1-\frac{s}{S}\right) \frac{\partial}{\partial t} G_{1}(T, s) d t
$$

where

$$
G_{1}(T, S):=\int_{0}^{T} \int_{0}^{S}\left(1-\frac{t}{T}\right) f(t, s) d t d s .
$$

It follows from (4.12), (4.13), and (4.14) that $\frac{\partial}{\partial S} G_{1}(T, S)$ is $(C, 0,1)$ integrable to $L$. By (4.12), we have

$$
G(T, S)=G_{1}(T, S)-H_{1}(T, S),
$$

where

$$
H_{1}(T, S)=\frac{1}{S} \int_{0}^{S} s \frac{\partial}{\partial s} G_{1}(T, s) d t .
$$

We have to show that $H_{1}(T, S) \rightarrow 0$ as $T, S \rightarrow \infty$.

By (4.13), we find

$$
\frac{\partial}{\partial S} G(T, S)=\frac{1}{S^{2}} \int_{0}^{S} s \frac{\partial}{\partial s} G_{1}(T, s) d s=\frac{H_{1}(T, S)}{T}
$$

We also have

$$
\begin{aligned}
\int_{S_{1}}^{S_{2}} \frac{\partial}{\partial S} G(T, S) d S & =G\left(T, S_{1}\right)-G\left(T, S_{2}\right) \\
& =\int_{S_{1}}^{S_{2}} \frac{H_{1}(T, S)}{S} d T \\
& =\int_{\log S_{1}}^{\log S_{2}} H_{1}\left(T, e^{v}\right) d v \\
& =\int_{\log S_{1}}^{\log S_{2}} R(T, v) d v
\end{aligned}
$$


Here, we used the substitution $S=e^{v}$ and $R(T, v)=H_{1}\left(T, e^{v}\right)$. We need to show that $\lim _{v \rightarrow \infty} R(T, v)=0$. By the simple calculation, we have

$$
\frac{\partial}{\partial v} R(T, v)=e^{v} \frac{\partial}{\partial v} H_{1}\left(T, e^{v}\right)=S \frac{\partial}{\partial S} H_{1}(T, S) .
$$

By (4.16), we get

$$
S H_{1}(T, S)=\int_{0}^{S} s \frac{\partial}{\partial s} G_{1}(T, s) d s .
$$

Differentiation the both sides of (4.19) with respect to $T$ gives

$$
H_{1}(T, S)+S \frac{\partial}{\partial S} H_{1}(T, S)=S \frac{\partial}{\partial S} G_{1}(T, S) .
$$

Taking the $(C, 1,0)$ mean of the both sides of (4.11) we have

$$
S \frac{\partial}{\partial S} G_{1}(T, S)=O(1),
$$

which implies that

$$
S \frac{\partial}{\partial S} H_{1}(T, S)=O(1)
$$

by (4.18).

We can easily obtain $H_{1}(T, S) \rightarrow 0$ as $T, S \rightarrow \infty$ by following the steps of Theorem 3.2 in [7]. It follows from (4.12) and (4.15) that $G_{1}(T, S) \rightarrow L$ as $T, S \rightarrow \infty$.

Since $G_{1}(T, S) \rightarrow L$ as $T, S \rightarrow \infty$ and the condition (4.10), we have $\lim _{T, S \rightarrow \infty} F(T, S)=L$ by Theorem 2 .

Remark 1. Analogous Tauberian results were proved in [8] for double improper integrals with a different perspective.

\section{CONCLUSiON}

In this paper, we extended the classical Tauberian theorems given for the $(C, \alpha)$ integrability of the improper integrals of functions of one variable to those of the $(C, \alpha, \beta)$ integrability improper integrals of functions of two by using the methods employed in Laforgia [7]. The analogous results for the functions of several variables can be obtained by the similar techniques.

\section{REFERENCES}

[1] J. Boos, Classical and modern methods in summability. New York, NY: Oxford University Press, 2000.

[2] I. Çanak and U. Totur, "A Tauberian theorem for Cesáro summability of integrals." Appl. Math. Lett., vol. 24, no. 3, pp. 391-395, 2011, doi: 10.1016/j.aml.2010.10.036.

[3] I. Çanak and U. Totur, "Tauberian conditions for Cesàro summability of integrals." Appl. Math. Lett., vol. 24, no. 6, pp. 891-896, 2011, doi: 10.1016/j.aml.2010.12.045. 
[4] I. Çanak and U. Totur, "Alternative proofs of some classical type Tauberian theorems for the Cesàro summability of integrals." Math. Comput. Modelling, vol. 55, no. 3-4, pp. 1558-1561, 2012, doi: 10.1016/j.mcm.2011.10.049.

[5] I. Çanak and U. Totur, "The $(C, \alpha)$ integrability of functions by weighted mean methods." Filomat, vol. 26, no. 6, pp. 1209-1214, 2012, doi: 10.2298/FIL1206209C.

[6] M. Dik, "Tauberian theorems for sequences with moderately oscillatory control modulo." Math. Morav., vol. 5, pp. 57-94, 2001.

[7] A. Laforgia, “A theory of divergent integrals.” Appl. Math. Lett., vol. 22, no. 6, pp. 834-840, 2009, doi: 10.1016/j.aml.2008.06.045.

[8] F. Móricz, "Tauberian theorems for Cesàro summable double integrals over $\mathbb{R}_{+}^{2}$." Stud. Math., vol. 138 , no. 1 , pp. 41-52, 2000.

[9] F. Móricz and Z. Németh, "Tauberian conditions under which convergence of integrals follows from summability $(C, 1)$ over $\mathbb{R}_{+} . "$ Anal. Math., vol. 26, no. 1, pp. 53-61, 2000, doi: 10.1023/A:1010332530381.

Authors' addresses

Ü. Totur

Adnan Menderes University, Department of Mathematics, 09010 Aydin, Turkey

E-mail address: utotureadu.edu.tr

I. Çanak

Ege University, Department of Mathematics, 35100 Izmir, Turkey

E-mail address: ibrahim.canak@ege.edu.tr 